\title{
Tendencias de investigación en Geografía Económica. Estudio bibliométrico de la Web of Science (1920-2020)
}

\section{Research trends in Economic Geography. Bibliometric study of the Web of Science (1920-2020)}

\author{
María Pilar Alonso-Logroño \\ pilar.alonso@udl.cat 100000-0002-4327-623X \\ Departament de Geografia i Sociologia. Universitat de Lleida. \\ Plaza Víctor Siurana, 1. 25003 Lleida, España \\ Nuria Bautista-Puig \\ nbautist@bib.uc3m.es @ 0000-0003-2404-0683 \\ Department of Industrial Management, Industrial Design and Mechanical Engineering. \\ University of Gävle. Kungsbäcksvägen 47, 801 76, Gävle, Suecia. \\ Universidad Carlos III de Madrid. Calle Madrid, 26. 28903 Madrid, España
}

INFO ARTÍCULO

Recibido: 20-12-2021

Revisado: 18-01-2022

Aceptado: 19-01-2022

\section{PALABRAS CLAVE}

Análisis bibliométrico Geografía Económica

Web of Science

Topic modelling

\section{KEYWORDS}

Bibliometric analysis

Economic geography

Web of Science

Topic modelling

\begin{abstract}
RESUMEN
Una de las primeras tareas para abordar cualquier trabajo académico es revisar las publicaciones llevadas a cabo sobre el tema. En este artículo se presenta una metodología que permite analizar las tendencias científicas en una disciplina a través de un análisis bibliométrico. En concreto, se examina su utilidad para estudiar los trabajos en el campo de la Geografía Económica; para ello se ha elegido como base de datos para su análisis la Web of Science (WoS). Con un conjunto de 4,307 publicaciones se analizan varios indicadores bibliométricos como la evolución de la producción científica, los agentes involucrados (países y organizaciones) y temas abordados, con el objetivo de ver la trayectoria del volumen de trabajos y las especializaciones temáticas de la Geografía Económica desde 1920 a la actualidad.
\end{abstract}




\section{INTRODUCCIÓN}

El incremento de la producción científica y la facilidad que ofrecen las redes provoca que el investigador, al revisar el tema sobre el que va a trabajar, se tenga que enfrentar al problema de la masificación de información bibliográfica. Puede verse desbordado si no utiliza herramientas adecuadas a la hora de realizar una búsqueda de los materiales que precisa. Para solventar este problema se puede acudir a la bibliometría, disciplina que permite, mediante el uso de herramientas, la identificación de la producción científica de una determinada temática, un actor (análisis de las publicaciones de un determinado país o institución) u otros niveles de análisis (por ejemplo, centrándose en las publicaciones de una o varias revistas especializadas) (Broadus, 1987). A través de los análisis bibliométricos se pueden analizar y caracterizar las distintas disciplinas, obteniendo información relevante de su trayectoria, de los patrones de colaboración, del impacto o la evolución de las temáticas, entre otras dimensiones.

Cada día son más frecuentes los estudios bibliométricos para conocer en profundidad las diferentes disciplinas, así como la identificación precisa de su producción y sus características. En el caso de la materia geográfica, algunas de sus ramas han tenido ya acercamientos de este tipo de análisis, sometiendo a una revisión sus producciones científicas. Así, Wang \& Liu (2014) trabajaron en una exploración bibliográfica sobre los estudios rurales en Geografía Humana, también Gorraiz, Gumpenberg \& Glade (2016) analizaron cuatro ramas de Geografía (Geoecología; Geografía Social y Económica; Demografía y Geografía de la población; Geografía Económica), entre las conclusiones que obtienen destacan que estas ramas tienen unas estrategias de publicación muy heterogéneas e individuales, incluso en el mismo campo de investigación. En 2019 un trabajo de Zhu, Jin \& He (2019) analiza la producción científica en Geografía Económica, centrándose sobre todo en la búsqueda de la línea central que ha seguido esta disciplina, indicando su gran variedad temática. En 2018 Kanai, Grant \& Lam (2018) utilizaron los análisis bibliométricos para el estudio de las temáticas urbanas. En 2019 Atienza et al. publicaron un trabajo apoyado en 55 revistas de la Web of Science (WoS) que se centra en estudiar a través de estudios bibliométricos las relaciones temáticas entre la Geografía y la Economía y la interdisciplinariedad de sus revistas.

Otros trabajos analizan la internacionalidad de la disciplina geográfica. Gutiérrez y López-Nieva (2001) en un estudio de una selección de 19 revistas de Geografía Humana de la WoS, determinaron que la mayoría no tienen un perfil internacional, sino que está más fragmentada en comunidades nacionales. En esta misma línea, Müller (2021) concluye que los productores del conocimiento en Geografía son anglófonos, mientras que la consumidores son cada vez más multilingües, situación que ocasiona que se pierdan la diversidad de conceptos, temas, estilos o localizaciones epistémicas que deberían constituirse como el distintivo de cualquier disciplina.

Recientemente en España también se han publicado algunos trabajos bibliométricos que aportan una información sobre algunas temáticas específicas en Geografía, como el trabajo de Salvador y Escolano (2018) sobre la revistas de Geografía indexadas en la WoS; en un artículo de Ródenas, Seguí-Pons \& Ruiz Pérez (2021), se analiza una revista concreta, "Journal of Transport Geography" entre 1993 y 2020, mostrando cómo las herramientas bibliométricas también tienen utilidad para diferentes unidades de análisis, por ejemplo, de revistas. Así mismo, en el trabajo de Corral y Espejo (2020), sus autores realizan un estudio de los artículos publicados en la revista Cuadernos Turísticos. En cualquier caso, no son muchos los análisis que encontramos desde una perspectiva bibliométrica en la disciplina geográfica, cuando realmente dada la variedad de temáticas y metodologías que se recogen detrás de ella, resulta necesario profundizar en esa diversidad interna que recogen sus contribuciones.

En este contexto, el objetivo de este artículo es presentar la utilidad de los análisis bibliométricos, para ello se toma el ejemplo de la producción científica de la Geografía Económica, dada la pluralidad temática que caracteriza a esta rama de la Geografía (Rosenman et al., 2020). Resulta útil acercarse a las técnicas que ofrece la bibliometría a la hora de ver resultados del estado de la cuestión del tema a abordar, y al que este trabajo intenta contribuir.

La estructura del artículo es la siguiente, tras la introducción, la sección 2 presenta la reflexión sobre la irregular trayectoria de la Geografía Económica y la necesidad de análisis que ayuden a aclarar su trayectoria, 
la sección 3 presenta la metodología de investigación 3. La sección 4 presenta los principales resultados. Finalmente, la sección 5 es el apartado de discusión y conclusiones que se derivan del análisis realizado.

\section{LOS CAMBIOS EN LOS ENFOQUES Y TEMÁTICAS DE LA GEOGRAFÍA ECONÓMICA}

En muchas disciplinas su trayectoria y enfoques son bastante lineales, ajustándose a una sucesión de paradigmas en cuanto a sus objetivos de estudio. En el caso de la Geografía vemos que los distintos cambios metodológicos han generado una evolución interna bastante convulsa, donde no es tan fácil ver una ruptura entre temas y metodologías. La Geografía ha pasado de ser la disciplina descriptiva del territorio, a ser una ciencia analítica y reflexiva, pero sin olvidar sus orígenes. Sin duda, las etapas que le ha tocado pasar han llevado a la Geografía a plantear métodos de trabajo nuevos, a acercarse a temáticas novedosas, que dieran respuestas a los problemas territoriales que una sociedad cambiante motivaba a realizar (Unwin, 1992).

Esta situación de diversidad que ha ido viviendo la Geografía plantea una necesidad de acercarse a las temáticas que los distintos trabajos han abordado y que las diferentes ramas de esta disciplina han ido abriendo a lo largo del tiempo. Es necesario tener presentes las aportaciones que se han ido realizando desde la Geografía e ir centrando las temáticas y las formas de trabajar a la hora de buscar aquellos trabajos que resulten útiles en las tareas a trabajar. Por ello, las aportaciones que se hagan sobre los análisis de la trayectoria de cuestiones y métodos que se han desarrollado a lo largo del tiempo son particularmente útiles en este campo de trabajo, donde a veces la diversidad puede parecer un caos, cuando realmente de ese caos surge la razón de la Geografía, el territorio y sus cambios.

La Geografía se ha visto en su trayectoria muy influida por los distintos cambios de paradigmas de la ciencia, planteados por Kuhn (1970), quien proporcionó una piedra angular en la discusión de las revoluciones de paradigmas. Destacó que un paradigma se crea cuando un individuo o grupo produce una síntesis o teoría que aporta más beneficios, tiene nuevas soluciones para los problemas y puede atraer a la mayoría de los científicos y profesionales de la próxima generación y alienta a los científicos a ver su participación en la investigación de manera diferente. Esto conducirá gradualmente a la desaparición de paradigmas anteriores, ya que el nuevo paradigma tiene más éxito que los anteriores. No obstante, el nuevo paradigma se enfrentará a desafíos y habrá científicos que se adherirán al viejo paradigma obstinadamente y, en muchos casos, desalientan la adopción de un nuevo paradigma. Sin embargo, habrá disciplinas, como la Geografía, en las que la convivencia y el pluralismo sea las que las defina y terminen definiéndola (Rosenman et al., 2020).

En este contexto, en este artículo se toma una de las ramas de la Geografía, la Geografía Económica, para comprobar esa convivencia y pluralismo, ya que es una de las disciplinas que ha ido pasando en su trayectoria por enfoques muy diversos en el tratamiento de los distintos temas que ha ido trabajando (Sánchez-Hernández, 2021). Los aportes que se han ido realizando en la Geografía Económica se han ajustado a los distintos paradigmas científicos, asumiendo los cambios en los intereses temáticos, en los modelos de acercamientos a los problemas planteados y también a las posibles soluciones ofertadas.

Pero, en esta disciplina, la ruptura de los distintos paradigmas científicos no es radical, sino que se observa una coexistencia entre enfoques teóricos y metodológicos, que hace que internamente no existan unos cambios radicales, sino una suma y una convivencia de temáticas (Méndez, 1997). Esto complica las búsquedas de los estados de la cuestión de los temas que se abordan. En la trayectoria de esta rama geográfica a los temas clásicos se han ido sumando otros derivados de la adopción de los planteamientos metodológicos, y también como resultado de los propios cambios en los tipos de funcionamientos de los sistemas económicos imperantes. Aunque la Geografía Económica a veces pueda dar la imagen de una rama poco consolidada, por sus diversos ámbitos y centros de trabajo (Rosenman et al., 2020), pero en realidad esta situación debe considerarse como un enriquecimiento.

Es necesario tener siempre presente que la Geografía Económica es una rama que se ha ido redefiniendo, ajustándose no solo a los cambios de los distintos paradigmas científicos, sino también en sus adaptaciones ha estado muy condicionada por las diversas situaciones sociales, políticas y económicas que han ido aconteciendo. Los investigadores que han trabajado en ella han tenido que adecuarse a los cambios y 
de ahí el posible "caos" temático que pueda parecer que presenta esta rama, que no es más que el fruto del propio carácter dinámico en la relación entre los procesos económicos y el desarrollo de los territorios, así como sus problemáticas.

Por tanto, en la trayectoria de esta rama de la Geografía, no existe una evolución lineal, donde observar que tras un paradigma le sustituye otro, sino que hay una superposición de diferentes escuelas de pensamiento que van nutriéndola de perspectivas, metodologías y donde se van añadiendo temas de trabajo, pero sin olvidar los anteriores (Sánchez-Hernández, 2021). Van surgiendo nuevos campos a la vez que se mantiene el interés por los anteriores. Por ello, es necesario mantener en la perspectiva de análisis, los enfoques más tradicionales, pero también los más recientes.

Los principales enfoques de la Geografía Económica parten de su consolidación como disciplina científica a finales del siglo XIX coincidiendo con una serie de acontecimientos: el Congreso Internacional de Geografía en 1875 donde se instituyó como rama de la Geografía; la publicación en Gran Bretaña de primer manual de Geografía Económica, "Handbook of comercial geography” de Chisholm en 1889 o la institucionalización como disciplina universitaria en la Universidad de Cornell en EE. UU. en 1893. En esta primera etapa de la Geografía Económica institucionalizada, los análisis de cuestiones económicas empezaron a tener autonomía en los trabajos de los geógrafos. Las temáticas principales que se trabajan se apoyan en la descripción y en las corrientes imperantes del historicismo y del determinismo ambiental, que otorgaban un papel importante a la relación de la condiciones del medio físico con las ocupaciones de la población, pero siempre bajo la perspectiva de realización de inventarios de los recursos naturales vinculados con las actividades económicas con el determinismo ambiental (minería, agricultura, industrias,...). Los objetivos de las aportaciones de esta etapa se centraron en un proyecto de tipo ambiental, donde imperaba la relación entre recursos naturales y desarrollo económico (Sánchez-Hernández, 2021).

Pero, los años cincuenta del siglo XX, los análisis regionales apoyados en la descripción son dejados al margen por un grupo de geógrafos que empiezan a plantear una aproximación de tipo cuantitativo. En la reivindicación de esta postura se defiende el uso de teorías y modelos para explicar los problemas territoriales de la localización. En este planteamiento los geógrafos económicos se implican de manera importante, y buscan esta postura como base para explicar la localización y la organización espacial derivada de la actividad económica. La estadística se convierte en una de las herramientas principales que utilizan, frente a la observación que manejan los geógrafos más tradicionales. Sin embargo, el exceso en el uso de las matemáticas también tuvo su respuesta entre los geógrafos, que ven la necesidad de preocuparse por los problemas de la organización social y los recursos económicos, donde temas de segregación económica, de pobreza, de mercados laborales o de reestructuración de la actividad industrial, pasan a preocupar a los geógrafos que se engloban bajo el paradigma de la Geografía Radical.

También desde los años 1980 se asiste a movimientos donde la cultura se convierte en el centro de las explicaciones y aparecen temas como el género en los diferentes mercados laborales, las redes sociales para la formación de economías alternativas, paisajes económicos vinculados a las presencias de determinadas actividades, entre otros. La Geografía Económica sufrió un enriquecimiento en el que factores sociales, políticos o psicológicos se introdujeron en la explicación de los diferentes procesos. En la que se puede considerar la tercera de las fases de la trayectoria de la Geografía Económica, con todo el anterior bagaje de corrientes mencionado y a las que se van sumando en las últimas décadas nuevos proyectos como el proyecto estructuralista, en que las explicaciones de los procesos se centran en la búsqueda de la interrelación entre estructuras globales y trayectorias locales. A estas nuevas corrientes también se le suma el reencuentro de la Geografía Económica y la Regional, mediante una reformulación de su relación, y también los argumentos de la Geografía Económica Evolucionista, en el que interesa el paisaje económico (Sánchez Hernández, 2021), cuyos estudios se caracterizan por relacionar los fenómenos que ocurren en la economía con principios biológicos propios de las ciencias naturales.

Encontramos por tanto una adición de proyectos aparentemente heterogéneos que se pueden albergar bajo el paraguas de "nuevas geografías económicas", donde lo que cobra importancia es la explicación de factores y procesos económicos sobre el espacio cambiante, en donde se hablara del espacio bajo el paraguas de economías de mayor desarrollo (ganadores) y de espacios más atrasados (perdedores), que 
generaran desequilibrios territoriales, ya que los espacios ganadores estarán conectados por redes, frente a las regiones marginales que estarán excluidas. Preocupaciones asociadas a temas que se van sumando al quehacer geográfico, donde surgen unidades de análisis como los distritos industriales o la globalización organizada a partir de las multinacionales o la innovación, pero también otros temas como las prácticas económicas alternativas y bajo este paraguas siembre un proyecto ambiental que resulta clave (Sánchez Hernández, 2021).

Estas tres fases de perspectivas adoptadas desde la Geografía Económica han acumulado metodologías diversas que van desde técnicas cualitativas con entrevistas, observación participante o encuestas, así como temáticas variadas, que se han ido añadiendo y que están presentes en los estudios más actuales y que se pueden seguir a través de los estudios bibliométricos. La Geografía Económica no ha sido una rama que haya avanzado en paradigmas descartando lo anteriores, sino que ha ido enriqueciéndose, sumando perspectivas, en un proceso de hibridación (Sánchez Hernández, 2021), donde es necesario profundizar en lo hecho y en lo que se está haciendo dentro de esta rama del conocimiento para ayudar a comprender con su trayectoria el funcionamiento de la economía sobre los territorios.

En este contexto, la aproximación a la investigación en Geografía Económica, disciplina con una trayectoria bastante divergente en cuanto a sus aportaciones, exige tener conocimiento de lo que estudia, de cuáles son sus principales contenidos temáticos, de cómo están evolucionando. Por ello los estudios bibliométricos son una solución ya que permiten observar y definir cronologías y temáticas que se han ido trabajando en esta rama, que se presenta como una materia muy abierta, e incluso desordenada, en cuanto a los contenidos que ha ido abordando.

\section{METODOLOGÍA}

Para el acercamiento a la trayectoria temática de la Geografía Económica, se utilizó un enfoque bibliométrico, es decir un análisis cuantitativo de las producciones científicas. Los pasos que se siguieron para ello fueron los siguientes: (i) Formulación de una estrategia de búsqueda para identificar la producción científica sobre Geografía Económica; (ii) Recopilación de datos y procesamiento de información; (iii) Definición y análisis mediante el uso de indicadores bibliométricos.

(i) Formulación de una estrategia de búsqueda. Para esta fase se diseñó una táctica de búsqueda para la recuperación de la producción de la rama de Geografía seleccionada, indagando en los campos de título, resumen y palabras clave (campo TS). Para esta búsqueda, se utilizaron las bases de datos de la Colección principal de la Web of Science (WOS), es decir, la Science Citation Index (SCI), Social Science Citation Index (SSCI) y Arts and Humanities Citation Index (A\&HCI). Se consideraron todos los años que contienen estas bases y se incluyeron todas las tipologías documentales. A pesar de las limitaciones de esta base de datos (Gómez-Caridad \& Bordons, 1996), su elección permitió una recopilación de aquellos trabajos indexados en las revistas incluidas en dicha base. La estrategia utilizada fue la siguiente:

\section{- Geografía económica: TS="economic-geography" AND TS="econom* geography"}

Esta estrategia de búsqueda permitió identificar la literatura básica (core), es decir, aquellos documentos relacionados de forma explícita con la Geografía Económica presente en la base de trabajos elegida para este análisis (WoS). El motivo de la elección de esta base es porque es una base de datos multidisciplinar, formada por una amplia colección de bases de datos bibliográficas, citas y referencias de publicaciones científicas de cualquier disciplina del conocimiento, siendo una de las bases de referencia para este tipo de estudios (Salvador \& Escolano, 2018).

(ii) Recuperación de resultados científicos y procesamiento de información. En esta segunda fase se descargaron los datos de las publicaciones de la WoS obtenidos de la consulta (en formato *.txt) y se exportaron a una base de datos relacional creada con MySQL, en la cual se ingresaron todos los registros. 
(iii) Definición de indicadores bibliométricos. Se analizaron los siguientes indicadores para el conjunto recopilado:

a) Patrones de investigación:

- Evolución anual de la producción científica y tasa de crecimiento promedio acumulada (o CAGR) medida a través de la siguiente fórmula.

$$
C A G R=\left(\sqrt[n-1]{\frac{x_{n}}{x_{1}}}-1\right) * 100
$$

Dónde y son los valores de inicio y final del período estudiado.

- Producción por instituciones y países: valores absolutos e Índice de Actividad que evalúa el grado de especialización de un país en un determinado tema (Frame, 1977), representando la contribución porcentual de cada país a la producción total de WoS, en comparación con el porcentaje de contribución en el tema analizado. La fórmula se detalla a continuación:

$$
A I=\frac{G \cdot E c o n \cdot A c}{G . E c o n . \text { WoS }}, \frac{\text { Total produccion Ac }}{\text { Total produccion WoS }}
$$

Dónde:

G.Econ. Ac es el total producción total de un actor (país) sobre un tema (Geografía Económica) en la WoS;

G.Econ.WoS es la producción total en Web of Science en Geografía Económica $(n=4307)$;

Total producción Ac es la producción total de un actor (país) en WoS en el mismo período;

Total producción WoS es el número total de documentos en WoS durante el período ( $n=67.546 .640)$,

Para este análisis, se han considerado las instituciones normalizadas de la WoS. Los datos posteriormente fueron mapeados con el software ArcGIS.

- Patrones de colaboración (nacional / internacional / sin colaboración). La colaboración nacional ocurre cuando al menos dos instituciones del mismo país contribuyen en el trabajo; colaboración internacional cuando hay instituciones de más de un país, y sin colaboración significa que el autor/es es/son de una sola institución.

- Impacto. Este apartado recopila aquellos trabajos en Geografía Económica que han tenido un mayor impacto, medido en número de citas totales.

b) Especialización temática:

- Categorías Web of Science (WoS). Este análisis permite mostrar un esquema de categorización de diferentes áreas de conocimiento de la WoS'1. Todos los libros y revistas incluidos en la colección principal de esta base de datos se asignan al menos a una de estas categorías. Las áreas de investigación se dividen en cinco grandes categorías (Artes y Humanidades; Ciencias de la Vida y Biomedicina; Ciencias Físicas; Ciencias Sociales; Tecnología) que a su vez se dividen en múltiples subáreas de investigación.

- Definición de temas mediante técnica de topic modelling, mediante los títulos y los abstracts de las publicaciones de los documentos recopilados mediante la estrategia de búsqueda realizado. Esta técnica probabilística permite identificar y aprender "temas" de un corpus de documentos.

1 Áreas de la Web of Science: http://images.webofknowledge.com/WOKRS522_2R1/help/es_LA/WOS/hp_research_areas_easca.html 
Con esta metodología cada documento es entendido como un conjunto de palabras (uni-gramas) que pueden combinarse en conjuntos o sacos de palabras (bags of words o topics) formados por aquellas que aparezcan más juntas de manera más frecuente (mayor co-ocurrencia). Mediante la selección de un umbral de probabilidad, cada documento se encuadra en uno o varios temas. Mediante este análisis un tema se define como una distribución de probabilidad sobre un vocabulario, donde las palabras con una alta probabilidad para el mismo tema tienden a tener una coocurrencia frecuente en los mismos documentos. Para realizar este análisis, primero se procedió a una depuración de los datos (eliminación de stopwords, espacios, etc.) mediante el software R (R Core Team 2020) y el uso del paquete tm (Feinerer \& Hornik, 2020). Para el análisis del topic modelling, se entrenó el dataset mediante el modelo Latent Dirichlet Allocation (LDA) en Python y se decidió utilizar un total de 15 clústeres de temas ya que este valor facilitaba la interpretación de los resultados. Además, se analizó la intensidad de los temas del período 1980 a 2020, para observar los cambios más recientes.

- Mapa de coocurrencias de palabras clave utilizando la herramienta VOSviewer (Van Eck y Waltman, 2017) para identificar grupos temáticos dentro del panorama científico. El tamaño de los nodos del mapa muestra la frecuencia de cada uno de los keywords obtenidos, los enlaces (links) las relaciones de co-ocurrencia entre dos términos (cuantas más veces aparecen juntos, tienen una conexión más grande) y los clústers (colores) indican la afinidad de los temas.

- Identificación de los Hot topics. Este tipo de análisis permite ver cuáles han sido los temas que han llamado más la atención en la literatura científica (medido por el aumento repentino del número de citaciones) utilizando el algoritmo de Kleinberg (2003). Para su identificación en el dataset de Geografía Económica se utilizó el software CiteSpace².

Explorar todos estos aspectos, a través de la base de datos de WoS, permite comprender mejor la configuración de la diversidad temática de la Geografía Económica, ver cuáles han sido las líneas más citadas, las instituciones que han aportado más trabajos de esta disciplina, etc. Con esta aproximación se quiere comprobar la utilidad de los estudios bibliométricos para conocer todos estos aspectos de referencia sobre las aportaciones científicas de una disciplina, como la Geografía Económica, que como se ha comentado, es cambiante y donde la hibridación de los proyectos científicos la caracteriza (Sánchez Hernández, 2021).

\section{RESULTADOS}

\subsection{Patrones de investigación}

Tras la consulta, descarga y análisis de la producción científica de Geografía Económica indexada en la WoS se identificaron 4.307 documentos en el período 1920-2020, con un crecimiento acumulativo medio de $8.12 \%$ (figura 1). En cuanto a la evolución de la producción científica, esta disciplina acumula un gran número de publicaciones a partir de los años 2000 (80,8\% de su producción). Sin duda, dentro de la explicación de esta trayectoria está la propia relevancia reciente alcanzada por las publicaciones en revistas indexadas en esta base de datos, que se ajustan a los requerimientos de evaluación de muchos concursos de promoción del profesorado o para evaluar la calidad de las publicaciones de las instituciones académicas. Los investigadores, por presiones de los sitemas de evaluación de la investigación, empiezan a buscar las revistas contenidas en las bases de datos indexadas, y que evalúan el factor de impacto. En cualquier caso, la explicación de este incremento también está relacionado con el recuperado interés de los economistas regionales de la que ellos denominan “Nueva Geografía Económica” (Krugman, 1997) y cuya literatura justificativa también es muy prolífica entre las revistas de esta base.

2 http://cluster.cis.drexel.edu/ cchen/citespace/ 
En este sentido, aun siendo conscientes de que nuestra búsqueda puede tener un cierto sesgo por aquí, no invalida el objetivo del trabajo, ya que con el análisis de los topic modelling y los Mapa de coocurrencias, se llega a encontrar los temas de trabajo que esconden estos trabajos que en principio se definen tanto en títulos, palabras clave o resumen por la presencia de las palabras de los términos definidos en la estrategia utilizada. Todo ello justifica que Geografía Económica empieza a tener un peso en las aportaciones de esta base de manera más notable desde el comienzo del siglo XXI, progresión que ha ido al alza, registrando en 2020 el mayor número de aportaciones, con casi 300 documentos. Esto demuestra que estamos ante una disciplina en crecimiento en la producción científica, con un importante dinamismo en las dos primeras décadas del siglo XXI.

Además de comprobar el crecimiento de los trabajos publicados en esta base de datos es interesante saber cuáles son las instituciones a las que pertenecen los investigadores que aportan estos trabajos; así en la tabla 1 se muestra el top 10 de las instituciones más prolíficas en esta rama. La mayor parte de las organizaciones son Universidades del Reino Unido y Estados Unidos, aunque también encontramos instituciones de Países Bajos, Suecia o Canadá. Todos ellos son centros de alto nivel en cuanto a las producciones y concentración de investigadores. La primera institución académica con más número de trabajos de Geografía Económica que publica en la WoS es la University of London (Reino Unido) con más de 180 documentos, seguida de la University of Oxford (Reino Unido) con 93. Es de destacar el número de instituciones inglesas con trabajos publicados en la WoS, así de las 10 primeras 5 son del Reino Unido.

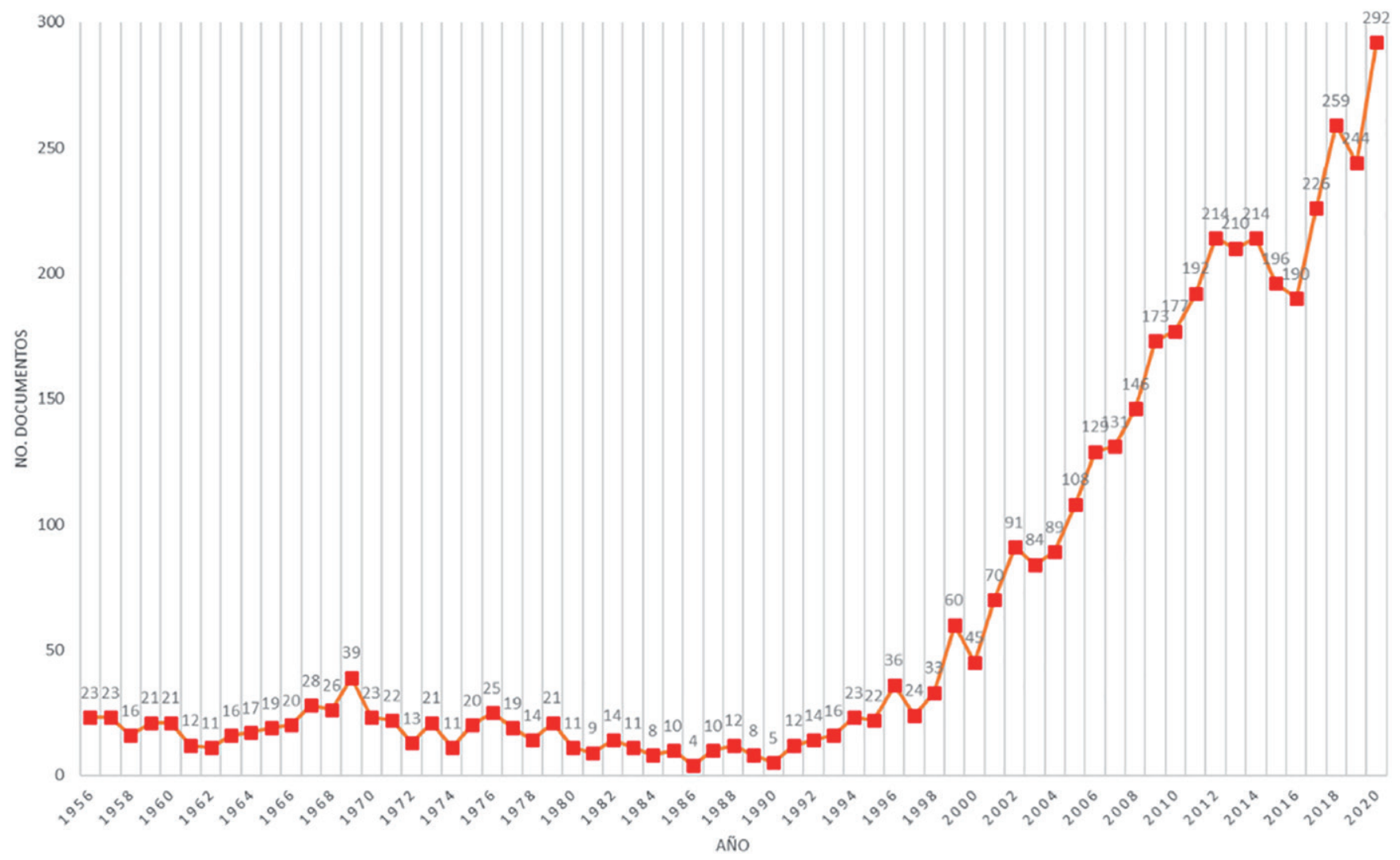

Figura 1. Distribución de publicaciones de Geografía Económica (1956-2020). Fuente: WoS. Elaboración propia. Nota: los cuatro documentos anteriores a 1956 no se muestran por ser discontinuos en el tiempo. 
Tabla 1. Top-10 instituciones en Geografía Economía.

\begin{tabular}{|l|c|}
\hline \multicolumn{1}{|c|}{ Institución } & G. Económica \\
\hline \multicolumn{1}{|c|}{ No. Docs. } \\
\hline University of London (Reino Unido) & 185 \\
\hline University of Oxford (Reino Unido) & 93 \\
\hline Utrecht University (Países Bajos) & 89 \\
\hline London School Economics Political Science (Reino Unido) & 85 \\
\hline University of California System (Estados Unidos) & 85 \\
\hline University of Cambridge (Reino Unido) & 73 \\
\hline Lund University (Suecia) & 67 \\
\hline University of Toronto (Canadá) & 65 \\
\hline National University of Singapore (Singapur) & 62 \\
\hline Newcastle University (Reino Unido) & 61 \\
\hline
\end{tabular}

Fuente: WoS. Elaboración propia.

Otra información que se obtiene del análisis realizado es la procedencia por países de los autores de los documentos analizados. Esta información se plasmó sobre un mapa donde se observa que países como Gran Bretaña, Estados Unidos, China o Alemania las que más número de documentos tienen publicados en el periodo analizado. Sobre todo, cabe destacar los dos primeros países, con más de 900 documentos cada uno. Sin embargo, al analizar por índice de actividad se observa una mayor especialización en el tema en el conjunto de sus aportaciones científicas en la WoS en comparación a las de otros campos en países como Noruega, Suecia, Finlandia, Chile, Eslovenia o Singapur (figura 2). España ocupa el lugar 21 del ranking por indice de actividad, lo que la sitúa en un campo de interes significativo, pero donde hay que mencionar la interrelación disciplinaria con las publicaciones de economistas espaciales, como más adelante se señala.

\subsection{Colaboración}

Si consideramos los patrones de colaboración de los autores que publican artículos relacionados con Geografía Económica, 898 documentos (20,8\%) son en colaboración internacional, 772 en colaboración nacional (17,2\%) y 2.637 sin colaboración (61.2\%). Si consideramos los últimos 20 años es la colaboración internacional la que ha crecido de forma más significativa (con un crecimiento de un 15,5\%), denotando una mayor colaboración con autores de ámbito internacional (figura 3), aunque el tipo de patrón predominante es el de autorías del mismo centro, con esa tendencia reciente apuntada, que en 2020 es bastante significativa. En esto quizás el aislamiento presencial y la mayor utilización de las redes para trabajar también puede ser un elemento explicativo.

\subsection{Impacto}

En términos de impacto, medido por el número de citas absolutas de los trabajos recopilados, la tabla 2 muestra los más altamente citados, con más de 1.000 citas. Lidera el ranking el trabajo de Krugman (1991) en el que desarrolla un modelo de análisis de los patrones de localización de actividades de centro-periferia en función de los costes de transporte y las economías de escala atendiendo a patrones de los procesos de industrialización. Por otro lado, le sigue el trabajo de Porter (1998) en el que habla de los clústeres y la economía de competición 
y Boschma (2005) con un análisis crítico de la proximidad espacial y la innovación. De los 7 trabajos tomados como ejemplo de temáticas de la cabecera con mayor índice de impactos, muestra la gran variedad de temáticas que se acogen dentro de la Geografía Económica.

De estos trabajos salvo 2 que están hechos con la implicación de geógrafos, el resto son investigadores de otras disciplinas con lo cual hay que valorar que no todos los trabajos aportados en el patrón de búsqueda están planteados desde la disciplina geográfica. Pero también hay que valorar la interrelación de las materias afines, y para la Geografía Económica la Economía lo es, ya que lo importante es ver las temáticas que bajo este paraguas preocupan a la sociedad y que son los que se abordan en todos estos trabajos.

Junto con los autores más citados también es de interés saber cuáles son las revistas más utilizadas para presentar los trabajos obtenidos mediante la estrategia de búsqueda realizada. Para ello la tabla 3 representa a las revistas con más de 50 documentos. Es de destacar que la mayoría de las revistas ocupan los primeros cuartiles del ranking del Journal Citation Reports (edición 2020), en el área de Geografía, lo que está indicando que se encuentran en revistas de relevancia dentro de su ámbito de conocimientos si se atiende a la citación de los trabajos de estas revistas.
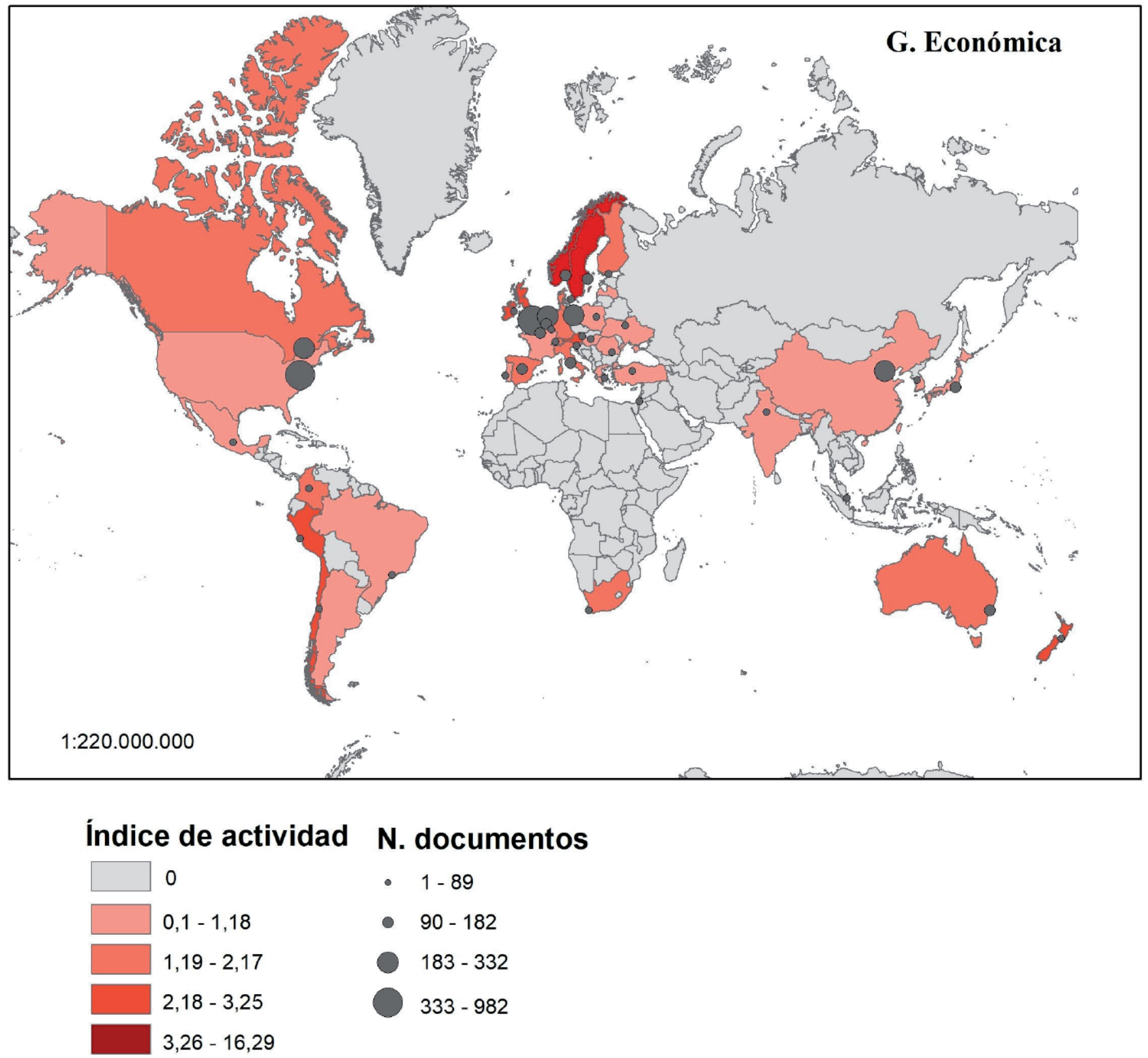

Figura 2. Distribución de la producción científica de G. Económica (>5 docs.) e Índice de Actividad 1920-2020. Fuente: WoS, ArcGis. Elaboración propia. 


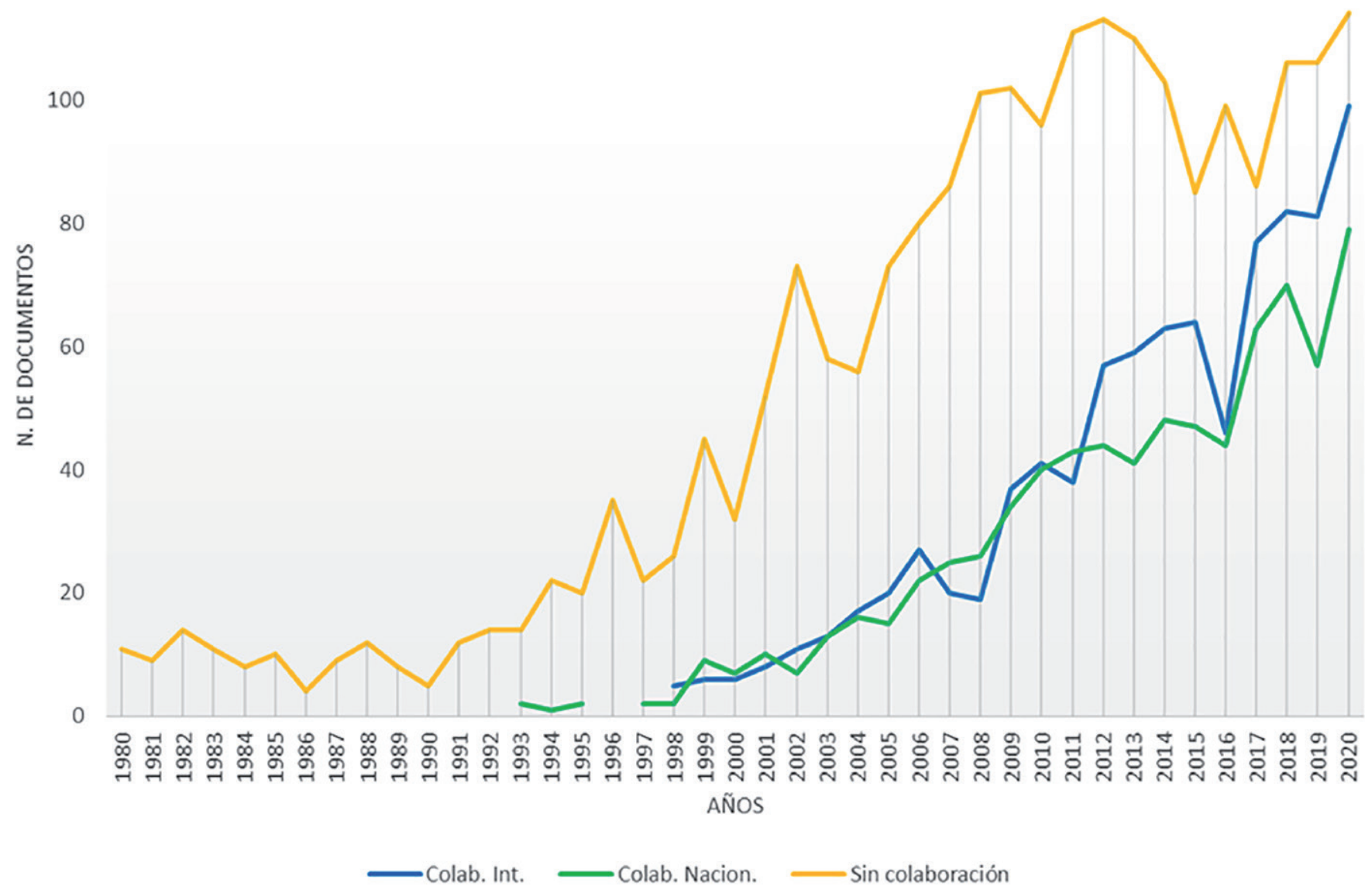

Figura 3. Evolución de los documentos en colaboración (nacional, internacional y sin colaboración) de 1980 a 2020. Fuente: WoS. Elaboración propia.

Tabla 2. Artículos altamente citados en Geografía Económica (>1000 citas).

\begin{tabular}{|l|c|}
\hline \multicolumn{1}{|c|}{ Trabajo } & \multicolumn{1}{|c|}{ Número de citas } \\
\hline $\begin{array}{l}\text { Krugman, P. (1991). Increasing returns and economic geography. Journal of } \\
\text { political economy, 99(3), 483-499. }\end{array}$ & 3.877 \\
\hline $\begin{array}{l}\text { Porter, M. E. (1998). Clusters and the new economics of competition (Vol. } \\
\text { 76, No. 6, pp. 77-90). Boston: Harvard Business Review. }\end{array}$ & 2.960 \\
\hline $\begin{array}{l}\text { Boschma, R. (2005). Proximity and innovation: a critical assessment. } \\
\text { Regional studies, 39(1), 61-74. }\end{array}$ & 1.857 \\
\hline $\begin{array}{l}\text { Porter, M. E. (2000). Location, competition, and economic development: } \\
\text { Local clusters in a global economy. Economic development quarterly, 14(1), } \\
\text { 15-34. }\end{array}$ & 1097 \\
\hline $\begin{array}{l}\text { Markard, J., Raven, R., \& Truffer, B. (2012). Sustainability transitions: An } \\
\text { emerging field of research and its prospects. Research policy, 41(6), 955- } \\
\text { 967. }\end{array}$ & 1.112 \\
\hline $\begin{array}{l}\text { Owen-Smith, J., \& Powell, W. W. (2004). Knowledge networks as channels } \\
\text { and conduits: The effects of spillovers in the Boston biotechnology } \\
\text { community. Organization science, 15(1), 5-21. }\end{array}$ & \\
\hline $\begin{array}{l}\text { Martin, R., \& Sunley, P. (2006). Path dependence and regional economic } \\
\text { evolution. Journal of economic geography, 6(4), 395-437. }\end{array}$ & 1.085 \\
\hline
\end{tabular}

Fuente: WoS. Elaboración propia. 
Tabla 3. Revistas donde más trabajos se publican (> 50 publicaciones). Entre paréntesis se indica el cuartil de cada revista según el JCR 2020.

\begin{tabular}{|c|c|c|}
\hline Revista & Número de documentos & $\%$ \\
\hline Journal Of Economic Geography (1Q) & 239 & 5,55 \\
\hline Economic Geography (1Q) & 224 & 5,20 \\
\hline Environment and Planning A-Economy And Space $(2 \mathrm{Q})^{*}$ & 176 & 4,09 \\
\hline Regional Studies (1Q) & 174 & 4,04 \\
\hline European Planning Studies (2Q) & 120 & 2,79 \\
\hline Tijdschrift Voor Economische En Sociale Geografie (2Q) & 105 & 2,44 \\
\hline Geoforum (1Q) & 101 & 2,35 \\
\hline Progress In Human Geography (1Q) & 97 & 2,25 \\
\hline Professional Geographer (3Q) & 88 & 2,04 \\
\hline Geography (2Q) & 79 & 1,83 \\
\hline Journal Of Regional Science (2Q) & 78 & 1,81 \\
\hline Regional Science And Urban Economics(2Q) & 72 & 1,67 \\
\hline Papers In Regional Science (2Q) & 70 & 1,63 \\
\hline $\begin{array}{l}\text { Annals Of The Association Of American Geographers (sin } \\
\text { determinar) }\end{array}$ & 64 & 1,53 \\
\hline Annals Of Regional Science (2Q) & 57 & 1,49 \\
\hline Zeitschrift Fur Wirtschaftsgeographie (3Q) & 54 & 1,32 \\
\hline
\end{tabular}

Fuente: WoS. Elaboración propia.

Nota: la Revista Environment and Planning A-Economy And Space engloba su producción, así como la antigua denominación de la revista (Environment And Planning A, EPA).

\subsection{Especialización por categorías de áreas de revista}

La Geografía Económica centra su producción en la categoría WoS de 'Geografía' (2.454 documentos, 56,98\%), así como Economía (1.876 documentos, 43,56\%) (figura 4). La siguiente categoría es 'Estudios Medioambientales' así como los temas urbanos, denotando el interés por el medio ambiente y el foco en los núcleos urbanos en la producción científica de esta rama. Esto sugiere que son temas que abordan la interacción entre el espacio y la economía.

\subsection{Topic modelling}

La tabla 4 resume los 15 clústeres de temas identificados mediante técnicas de topic modelling de la producción científica en Geografía Económica. En estas agrupaciones de palabras que se agrupan en distintos trabajos, se observa cómo unos temas son más sobre mercados/comercios (Topic 0, Topic 2, Topic 4), otros se centran más en espacios rurales (Topic 8 ) o urbanos (Topic 6, Topic 9) o incluso en temas de emprendimiento (Topic 14). Sin embargo, tal y como se observa en la figura 5 que muestra la intensidad de esos clústers en los últimos cuarenta años (período 1980-2020), no todos los temas han atraído el mismo interés. Temas espaciales (por ejemplo, regiones en vías de desarrollo como África (Topic 11) o China (Topic 6) o el emprendimiento (Topic 14) tuvieron más relevancia en los años 80-90; sin embargo, el análisis institucional o incluyendo a la sostenibilidad (Topic 5) o el transporte (Topic 4) han cobrado más relevancia recientemente, a partir del año 2000. 

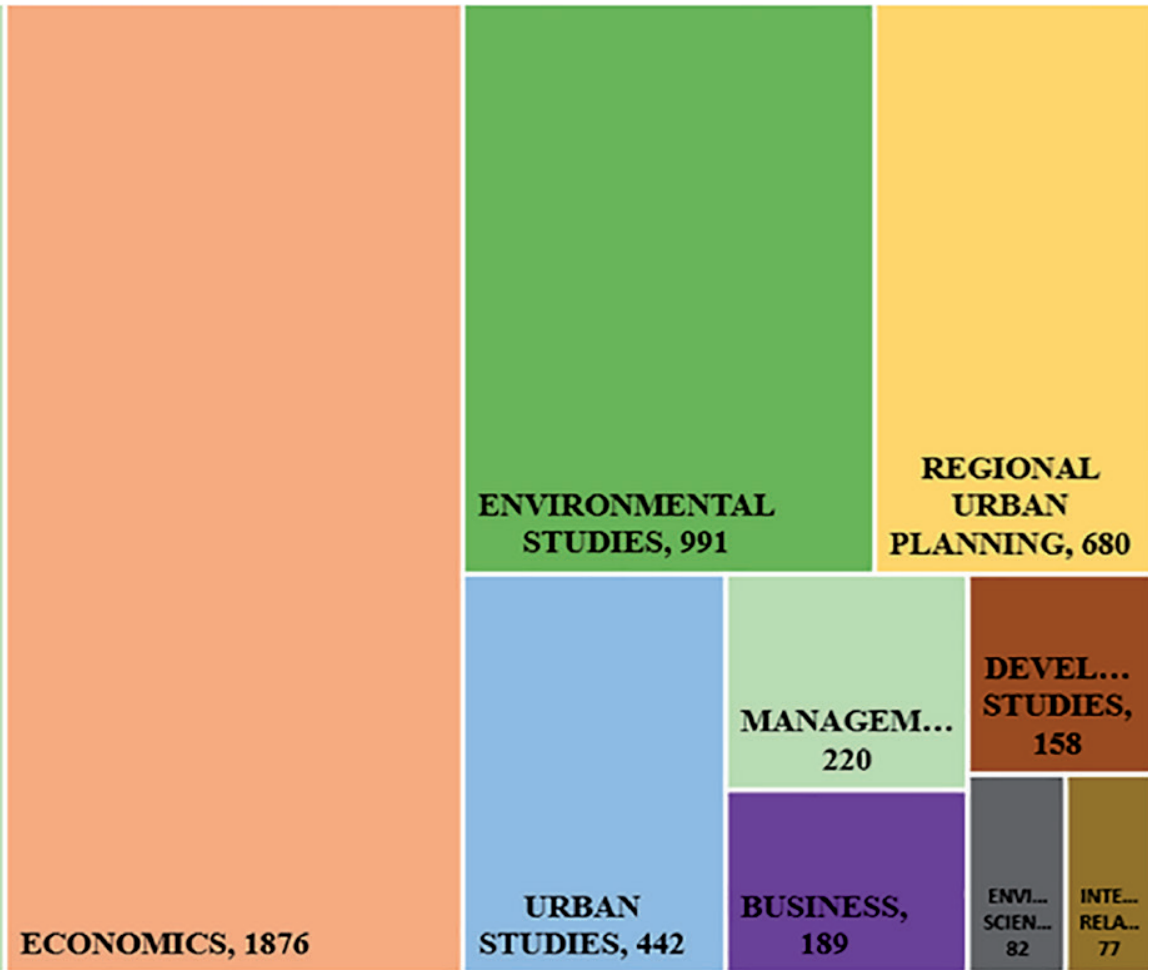

Figura 4. Distribución de Geografía Económica por categorías WoS. Fuente: WoS, Elaboración propia.

Un análisis pormenorizado de los clústers, permite por ejemplo mostrar cómo en la década de los 1990, hubo un importante número de trabajos centrados en los cambios de localización industrial asociados al crecimiento chino (Topic 6), o como los temas más de recursos medioambientales preocuparon en los 1980 (Topic 14), pero disminuyen su interés en las décadas siguientes.

La figura 6 muestra los mapas de coocurrencia de palabras clave para identificar los temas de interés de cada una de las disciplinas. El tamaño de los nodos del mapa muestra la frecuencia de cada uno de los keywords obtenidos, los enlaces (links) las relaciones de coocurrencia entre dos términos (cuantas más veces aparecen juntos, tienen una conexión más grande) y los clústers (colores) indican la afinidad de los temas. Al analizar los últimos 40 años, se observa que ha habido un cambio de interés en las temáticas. En un primer período predominaba el tema de los mercados y de la actividad económica en general, en un período más reciente se han incluido aspectos como la gobernanza, las redes o la innovación (figura 6, tabla 5).

Tabla 4. Resumen de los temas identificados por topic modelling.

\begin{tabular}{|l|l|}
\hline \multicolumn{1}{|c|}{ Temas } & \multicolumn{1}{c|}{ Conceptos } \\
\hline Topic 0 & $\begin{array}{l}\text { Firma; comercio; región; efecto; aglomeración; industria; productividad; regional; país; artículo; } \\
\text { resultado; modelo; crecimiento; nivel; fabricación; alto; espacial; evidencia; rendimiento; factor }\end{array}$ \\
\hline Topic 1 & $\begin{array}{l}\text { Conocimiento; innovación; red; firma; grupo; investigar; local; industria; proximidad; social; formación; } \\
\text { artículo; proceso; rol; diferente; tecnológico; negocio; creación; ajustado; dato }\end{array}$ \\
\hline Topic 2 & $\begin{array}{l}\text { Ubicación; empresa; ambiental; gas; negocio; mne; aceite; multinacional; investigar; política; firma; } \\
\text { energía; empresa multinacional; extranjero; análisis; estrategia; emisión; ruso; Rusia; inversión }\end{array}$ \\
\hline Topic 3 & $\begin{array}{l}\text { Trabajador; modelo; turismo; isla; impacto; renovable; movilidad; base; espacial; resultado; lugar de } \\
\text { trabajo; elección; labor; región; caso; variable; centrar; viaje; pérdida; ingreso }\end{array}$ \\
\hline Topic 4 & $\begin{array}{l}\text { Modelo; mercado; costo; comercio; transporte; efecto; sueldo; aglomeración; economía; espacial; papel; } \\
\text { aumento; país; precio; calificar; ubicación; casa; acceso; coste de transporte; competencia }\end{array}$ \\
\hline
\end{tabular}




\begin{tabular}{|c|c|}
\hline Temas & Conceptos \\
\hline Topic 5 & $\begin{array}{l}\text { Grupo; desarrollo; enfoque; artículo; social; investigar; industrial; economía; proceso; institucional; } \\
\text { artículo; trabajo; sostenibilidad; cambio; concepto; industria; política; sendero; perspectiva; práctica }\end{array}$ \\
\hline Topic 6 & $\begin{array}{l}\text { China; regional; espacial; desarrollo; sendero; ciudad; cambio; análisis; industrial; patrón; crecimiento; } \\
\text { papel; región; evolución; resultado; perspectiva; industria; nivel; Chino; aglomeración }\end{array}$ \\
\hline Topic 7 & $\begin{array}{l}\text { Firma; Servicio; inversión; extranjero; país; industria; impuesto; enlace; actividad; economía; exportar; } \\
\text { financiero; aglomeración; región; artículo; negocio; sector; internacional; empresa; ubicación }\end{array}$ \\
\hline Topic 8 & $\begin{array}{l}\text { Rural; periferia; ubicación; geográfico; área; recurso; subsidiario; industrial; espacial; planta; local; país; } \\
\text { modelo; actividad; región; concentración; sector; distrito; agricultura }\end{array}$ \\
\hline Topic 9 & $\begin{array}{l}\text { Ciudad; urbano; espacial; crecimiento; regional; efecto; artículo; hsr; desarrollo; modelo; migración; } \\
\text { impacto; China; local; europeo; derrame; resultado; accesibilidad; red; región }\end{array}$ \\
\hline Topic 10 & $\begin{array}{l}\text { Global; red; producción; cadena; valor; turismo; firma; internacional; investigar; gobernancia; } \\
\text { estratégico; local; valor global; mercado; perspectiva; producción global; artículo; gpn; enfoque; actor }\end{array}$ \\
\hline Topic 11 & $\begin{array}{l}\text { Africano; diversidad; mundo; papel; pueblo; desarrollo; futuro; Expresar; labor; Japonés; problema; } \\
\text { histórico; área; clase; movimiento; historia; regalo; cotidiano; población; trabajo }\end{array}$ \\
\hline Topic 12 & $\begin{array}{l}\text { Economía; creativo; financiero; investigar; industria; artículo; global; mercado; político; espacio; artículo; } \\
\text { cultural; red; práctica; geógrafo; industria creativa; análisis; espacial; comprender; trabajo }\end{array}$ \\
\hline Topic 13 & $\begin{array}{l}\text { Regional; innovación; política; desarrollo; región; sistema; industria; conocimiento; proceso; pah; papel; } \\
\text { desarrollo regional; diversificación; industrial; evolutivo; perspectiva; estudio; base; artículo; enfoque }\end{array}$ \\
\hline Topic 14 & $\begin{array}{l}\text { Alemán; emprendimiento; Banco; de emprendedor; bosque; Sur; recurso; deuda; mercado; empresario; } \\
\text { venta minorista; Alemania; recurso natural; lugar; este; natural; ecología; proceso; labor; norte }\end{array}$ \\
\hline
\end{tabular}

Fuente: WoS. Elaboración propia.

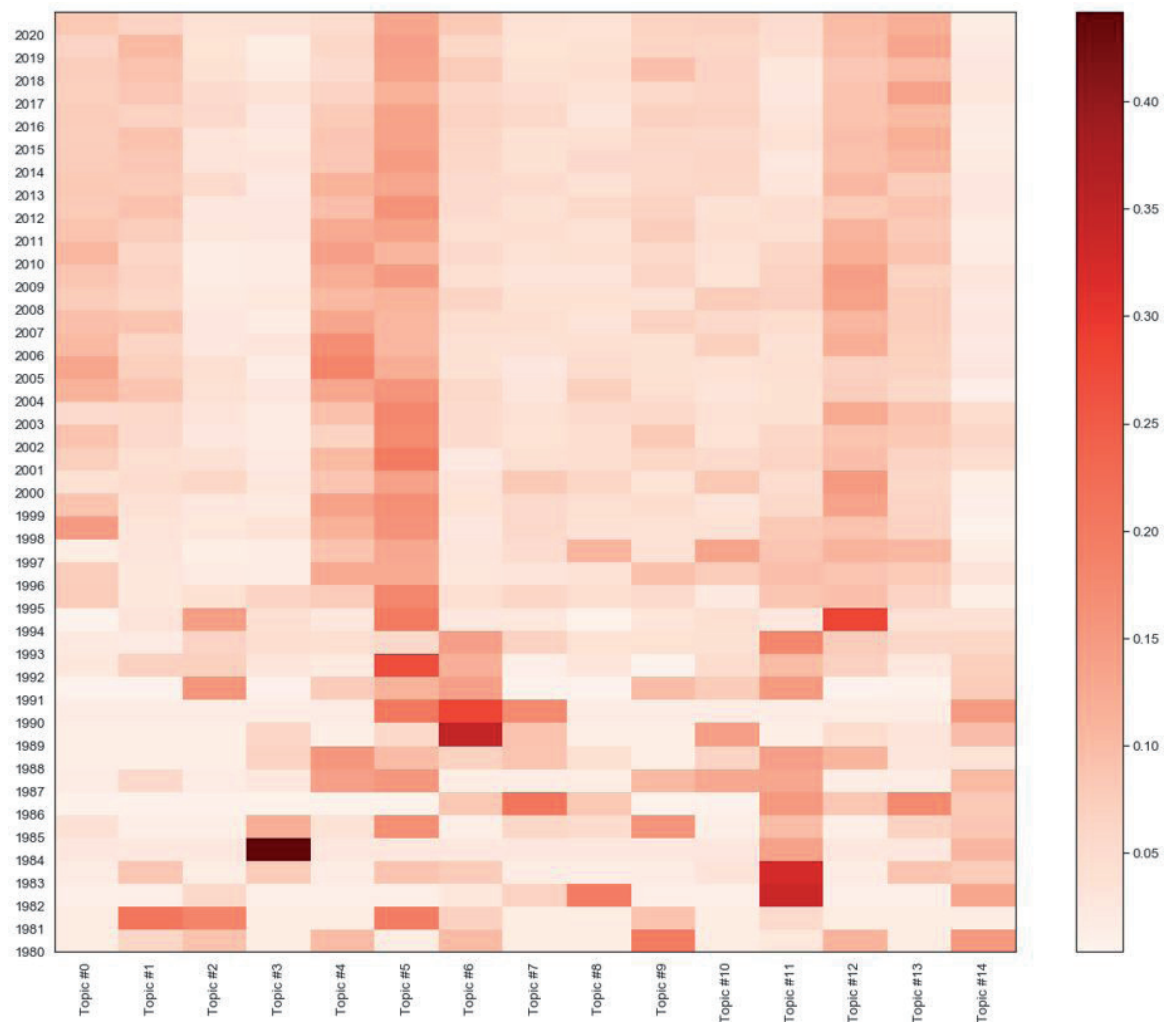

Figura 5. Evolución de la intensidad de los temas de 1980 a 2020. Fuente: WoS, elaboración propia. 


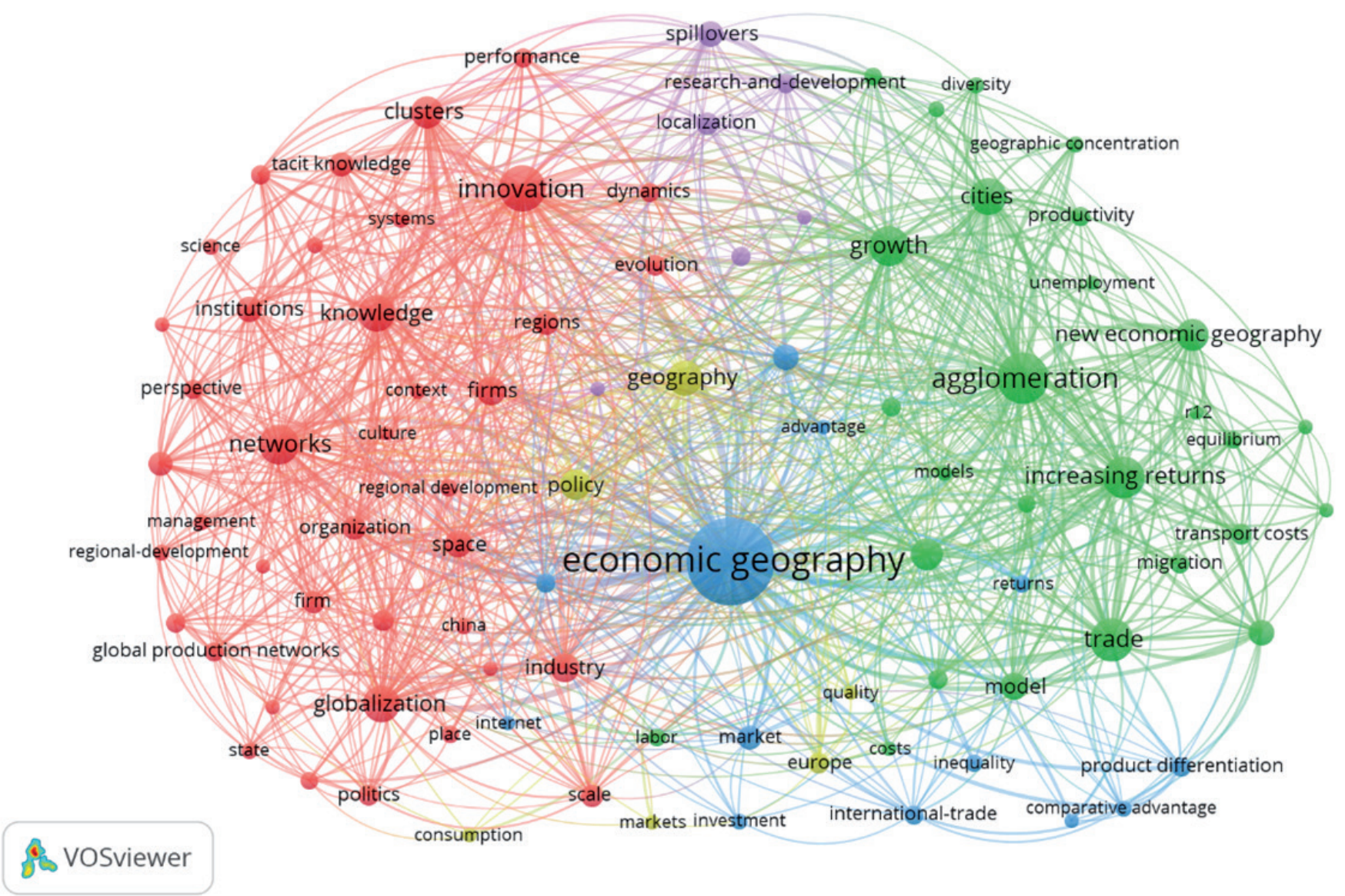

Figura 6. Mapas de coocurrencias de palabras clave de geografía económica en el período 2000-2010 (a) y 2010-2020 (b). Fuente: WoS, VOSViewer. Elaboración propia.

Tabla 5. Resumen de los clústeres obtenidos con los temas de interés.

\begin{tabular}{|c|c|c|c|}
\hline Geografía & $\mathrm{N}^{\circ}$ Cluster & Etiqueta & Términos más frecuentes \\
\hline \multirow{5}{*}{ Económica (2000-2009) } & \#1 (Rojo) & Redes e innovación & $\begin{array}{l}\text { Innovación (155); Redes (116); } \\
\text { Conocimiento (100) }\end{array}$ \\
\hline & \#2 (Verde) & Actividad económica & $\begin{array}{l}\text { Aglomeración (187); Comercio (134); } \\
\text { Rendimientos crecientes (128) }\end{array}$ \\
\hline & \#3 (Azul) & Inversión y mercado & $\begin{array}{l}\text { Geografía económica (551); } \\
\text { Competición (46); Mercado (40) }\end{array}$ \\
\hline & \#4 (Amarillo) & Políticas & Geografía (92); Política (66); Europa (30) \\
\hline & \#5 (Morado) & $\begin{array}{l}\text { Externalidades e } \\
\text { invetigación }\end{array}$ & $\begin{array}{l}\text { Derrames (45); Localización (39); } \\
\text { Investigación-Y-Desarrollo (29) }\end{array}$ \\
\hline \multirow{4}{*}{ Económica (2010-2020) } & \#1 (rojo) & Actividad económica & $\begin{array}{l}\text { Geografía económica (1211); } \\
\text { Crecimiento (279); Aglomeración (274) }\end{array}$ \\
\hline & \#2 (verde) & Gobernanza & $\begin{array}{l}\text { Globalización (156); Gobernanza (125); } \\
\text { Redes de producción globales (113) }\end{array}$ \\
\hline & \#3 (azul) & Redes e innovación & $\begin{array}{l}\text { Innovación (400); Conocimiento (281); } \\
\text { Redes (191) }\end{array}$ \\
\hline & \#4 (amarillo) & Dinámicas industriales & $\begin{array}{l}\text { Geografía (186); Dinámica (165); } \\
\text { Evolución (157) }\end{array}$ \\
\hline
\end{tabular}

Fuente: WoS, VOSViewer. Elaboración propia. 


\subsection{Hot Topics}

La tabla 6 muestra detección de ráfagas en las 35 palabras clave de todo el conjunto de datos trabajados sobre Geografía Económica. Sobre cada concepto, se indica la fuerza de su ráfaga, el período de inicio y fin de la ráfaga, además de la evolución temporal (en rojo el período y duración de la ráfaga). "Impacto" $(13,77)$, "nueva geografía económica" $(13,65)$, "aumento del rendimiento" $(13,4)$, "organización" $(12,43)$ y "comercio" $(11,78)$ son las palabras que tienen los valores de citación más altos. Sin embargo, si se comprueba temporalmente esta información, las palabras clave "organización" o "comercio internacional", tienen más importancia en los años 90 mientras que conceptos como "sostenibilidad", o "urbanización" han atraído más atención recientemente (desde 2018).

Table 6. Top-35 de las keywords con mayor ráfaga de citación.

\begin{tabular}{|c|c|c|c|c|}
\hline $\mathrm{Kw}$ & Fuerza & Inicio & Fin & $1956-2020$ \\
\hline Impact & 13,77 & 2016 & 2020 & \\
\hline New economic geography & 13,65 & 2005 & 2012 & \\
\hline Increasing return & 13,4 & 1998 & 2011 & \\
\hline Organization & 12,43 & 1994 & 2006 & \\
\hline Trade & 11,78 & 1998 & 2006 & \\
\hline Monopolistic competition & 10,3 & 1998 & 2009 & \\
\hline International trade & 8,93 & 1999 & 2006 & \\
\hline Dynamics & 8,92 & 2017 & 2020 & \\
\hline Transport cost & 8,84 & 2005 & 2010 & \\
\hline Resilience & 8,5 & 2014 & 2018 & \\
\hline Creation & 8,49 & 2017 & 2020 & \\
\hline Scale & 7,99 & 2001 & 2010 & \\
\hline Innovation system & 7,98 & 2016 & 2020 & \\
\hline Product differentiation & 7,89 & 2001 & 2009 & \\
\hline Global value chain & 7,68 & 2015 & 2020 & \\
\hline Path dependence & 7,66 & 2013 & 2017 & \\
\hline Localization & 7,54 & 2004 & 2010 & \\
\hline Offshore wind & 7,52 & 2016 & 2020 & \\
\hline Embeddedness & 7,14 & 2003 & 2008 & \\
\hline Power & 7,13 & 1999 & 2005 & 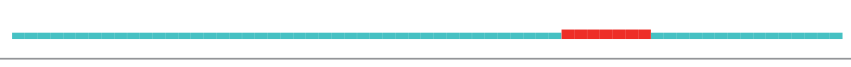 \\
\hline Return & 7,13 & 2005 & 2010 & \\
\hline Relatedness & 6,86 & 2016 & 2020 & \\
\hline Agglomeration & 6,63 & 2010 & 2011 & \\
\hline District & 6,59 & 1994 & 2003 & \\
\hline
\end{tabular}




\begin{tabular}{|c|c|c|c|c|}
\hline $\mathrm{Kw}$ & Fuerza & Inicio & Fin & $1956-2020$ \\
\hline Specialization & 6,54 & 2004 & 2010 & \\
\hline Equilibrium & 6,43 & 2005 & 2011 & \\
\hline Multinational enterprise & 6,36 & 2015 & 2018 & ـ \\
\hline Competition & 6,27 & 2002 & 2004 & - \\
\hline Manufacturing industry & 6,26 & 2010 & 2014 & \\
\hline $\begin{array}{l}\text { Evolutionary economic } \\
\text { geography }\end{array}$ & 6,26 & 2017 & 2020 & \\
\hline Path creation & 6,19 & 2018 & 2020 & - \\
\hline $\begin{array}{l}\text { Regional innovation } \\
\text { system }\end{array}$ & 6,13 & 2016 & 2020 & \\
\hline Flexibility & 6,11 & 1993 & 2003 & \\
\hline China & 6,09 & 2017 & 2020 & \\
\hline Life cycle & 6,08 & 2013 & 2018 & \\
\hline Dependence & 5,91 & 2014 & 2020 & \\
\hline Regional policy & 5,84 & 2009 & 2012 & \\
\hline Diversification & 5,84 & 2016 & 2020 & \\
\hline Institutional change & 5,82 & 2015 & 2018 & \\
\hline Value chain & 5,78 & 2013 & 2020 & \\
\hline Wage & 5,42 & 2005 & 2012 & \\
\hline Emergence & 5,42 & 2017 & 2020 & - \\
\hline Sustainability & 5,26 & 2018 & 2020 & \\
\hline Urban & 5,24 & 2018 & 2020 & \\
\hline Sustainability transition & 5,23 & 2016 & 2018 & 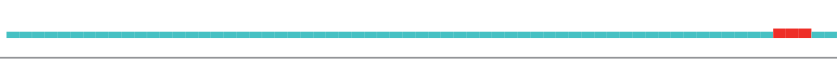 \\
\hline
\end{tabular}

Fuente: WoS. Elaboración propia.

\section{DISCUSIÓN Y CONCLUSIONES}

El análisis bibliométrico ofrece al investigador un punto de partida para abordar su investigación ya que le permite encontrar datos sobre la producción científica del tema que va a abordar. En este trabajo, tomado como ejemplo una disciplina que ha pasado por múltiples tendencias y temáticas como es la Geografía Económica, se intenta ver cómo dentro del pluralismo interno existen unas tendencias en las producciones científicas, que terminan reflejando las necesidades de análisis que implica una economía cambiante en los territorios. Esto permite ver que la diversidad temática que presenta una disciplina no supone que no tenga centros de trabajo que la definan, sino que los temas que preocupan a sus investigadores son cambiantes porque la economía lo es.

La Geografía Económica constituye una de las ramas de la geografía que, por su propio objetivo de estudio, las interrelaciones del funcionamiento de la economía y el territorio en diferentes escalas, provoca que los temas que abordan sean además de numerosos, cambiantes en enfoques y en preocupaciones. Sin 
embargo, no existen muchos estudios bibliométricos que analicen su producción científica, y las distintas temáticas de la propia disciplina, brecha de investigación que este estudio cubre.

El número de referencias encontrados en los criterios de búsqueda realizados son altos, a pesar de que la metodología utilizada es restrictiva, centrándose en el 'core' de las publicaciones y no se ha utilizado otras tipologías documentales como otros estudios que se centran en libros (Gorraiz et al., 2016) o en el análisis de la producción de las revistas en Geografía Económica (Foster et al., 2007). Además, los resultados obtenidos del número de publicaciones sugieren que a pesar de que la investigación sobre este tema parece tener una larga tradición, hay una concentración de publicaciones en los años más recientes, denotando un interés creciente sobre la Geografía Económica en los últimos años. A pesar que este crecimiento se encuentra generalizado en todas las áreas de conocimiento (Hu et al., 2020), este crecimiento está en línea con otros estudios (por ejemplo, Wang \& Liu (2014), que muestran que la parte económica es una gran contribuidora por ejemplo en los estudios de estudios territoriales como el ámbito rural.

El análisis realizado observa también una evolución positiva de los patrones de colaboración internacional, aunque estos siguen siendo menores, predominando las colaboraciones dentro de los mismos centros y dentro del mismo país (colaboración nacional). Este hecho reafirma las conclusiones de trabajos anteriores en las que se afirma que la Geografía Económica no tiene un carácter muy internacional (Gutiérrez \& López-Nieva, 2001 y Müller, 2021). También es interesante destacar la buena situación de las publicaciones analizadas en términos de impacto (por número de citas), lo que indica que los trabajos examinados están en revistas de las primeras posiciones de este ranking por citaciones, lo que sin duda favorece su mayor difusión. Sobre las temáticas abordadas mediante el análisis del topic modelling, los resultados obtenidos permiten ver como de variados son los temas y sobre todo lo que destaca es cómo hay momentos que interesan más unos que otros, así en los últimos años se aprecia como temas vinculados al transporte o la sostenibilidad están presentes en los trabajos analizados.

Por otro lado, los resultados muestran el carácter anglocéntrico (Reino Unido y Estados Unidos) de las instituciones que publican en geografía económica, en línea con estudios anteriores (Gutiérrez \& López-Nieva, 2001; Wing \& Liu, 2014; Foster et al., 2007) que muestran como se marginalizan a las comunidades no hablantes de inglés, a pesar de que la mayoría de lectores son consumidores son cada vez más multilingües (Müller, 2021). Sin embargo, este hecho puede estar relacionado con la selección de la base de datos WoS., que produce un sesgo hacía publicaciones en inglés. Sin embargo, los estudios por índice de actividad demuestran que otros países están especializados en el tema como los países nórdicos, han adquirido una especial importancia sobre todo porque las temáticas recientes asociadas a temas de innovación, emprendimiento han sido muy abundantes en estos países. Se asocian así temas de interés con espacios donde determinados procesos económicos presentan una novedad en su funcionamiento o en las preocupaciones sociales, algo que los resultados permiten mostrar.

En cualquier caso y como analizan Atienza et al. (2019), las interrelaciones temáticas entre los trabajos de los economistas y los geógrafos económicos resulta difícil de separar, como se ha podido comprobar en los resultados obtenidos con aportaciones de economistas incluidos en esta búsqueda. Si interesara separar los trabajos de ambos tipos de disciplina sería necesario en los criterios de búsqueda introducir algún discriminador que permita refinar la búsqueda si sólo queremos aportaciones realizadas desde el ámbito de los geógrafos. Aunque la interdisciplinariedad en este caso también ayuda a centrar cuáles son las temáticas que más preocupan a los investigadores que trabajen la interrelación entre economía y el espacio. Pero para este trabajo de acercamiento a los temas que se han abordado desde la geografía económica los patrones de búsqueda se consideran correctos, de hecho, los trabajos que aparecen como más citados son trabajos muy conocidos y utilizados en los trabajos de los geógrafos.

El trabajo realizado es una aproximación a la temática, en la que cabe señalar algunas limitaciones. Primero, identificamos los artículos relacionados explícitamente con la Geografía Económica, es decir, el 'núcleo' que incluyen tanto en el título, resumen como palabras claves los términos. En este sentido, puede haberse omitido artículos relacionados con el tema que no incluyan explícitamente la estrategia de búsqueda utilizada. Además, el uso de la base de datos WoS puede omitir otros trabajos no presentes en ellas, así como otra tipología de documentos (informes) que puedan resultar de interés para el análisis en la trayectoria de 
este campo. Un análisis más completo exigirá comprobar si esto mismo ocurre en otras bases bibliográficas donde se incorporen producciones en lenguas latinas, francesas e italianas, poco presentes en la base sobre la que se ha realizado esta aproximación.

Pero el objetivo era ofrecer una primera aproximación al interés de las técnicas de trabajo de la bibliometría cuando el investigador se enfrenta a campos de trabajo con una trayectoria compleja. En investigaciones futuras, se incluirán otras técnicas de búsqueda (métodos de expansión a través de citas directas), además de la definición de un marco teórico con un análisis basado en contenido de los artículos. Igualmente, en futuros estudios podrían complementarse con métodos de investigación cualitativa (entrevistas, encuestas) con investigadores del ámbito para conocer más sus motivaciones en la especialización en Geografía Económica. En cualquier caso, el método seguido demuestra la utilidad que tienen este tipo de acercamiento a las producciones científicas, y en definitiva la importancia de la interdisciplinariedad de las ciencias para ofrecer mejores resultados.

\section{Agradecimientos}

Se agradece el apoyo de financiación del proyecto "Innovación Socioeconómica y estrategias post-pandemia en los espacios metropolitanos de Valencia y Zaragoza" (PID2020-112734RB-C31), financiado por MCIN/ AEI/10.13039/501100011033/.

\section{Declaración responsable y conflicto de intereses}

Las autoras declaran que no existe ningún conflicto de interés en relación con la publicación de este artículo. Las autoras manifiestan que el presente artículo es fruto de un trabajo colaborativo y ambas han participado de forma equilibrada en la revisión bibliográfica, el diseño de la metodología, la realización del análisis gráfico y la redacción del artículo.

\section{REFERENCIAS}

Atienza, M., Ronda-Pupo, G. A., \& Phelps, N. (2019). Bridges over troubled water? Journals, geographers and economists in the field of economy and space 1980-2017. Environment and Planning A: Economy and Space, 51(8), 1800-1823. https://doi.org/10.1177/0308518X19866213

Broadus, R. N. (1987). Toward a definition of "bibliometrics". Scientometrics, 12(5-6), 373-379. https://doi.org/10.1007/ BF02016680.

Corral-Marfil, J. A. \& Espejo Marín, C. (2020). Evolución y análisis bibliométrico de la Revista Cuadernos de Turismo (19982019). Cuadernos de Turismo, (46), 531-565. https://doi.org/10.6018/turismo.451951

Feinerer, I, \& Hornik, K (2020). tm: Text Mining Package. R package version 0.7-8. https://CRAN.R-project.org/package=tm

Foster, J., Muellerleile, C., Olds, K., \& Peck, J. (2007). Circulating economic geographies: Citation patterns and citation behaviour in economic geography, 1982-2006. Transactions of the Institute of British Geographers, 32(3), 295-312. https://doi.org/10.1111/j.1475-5661.2007.00239.x

Frame, J. D. (1977). Mainstream Research in Latin America and the Caribbean. Interciencia, 2(3), 143-148. https://ci.nii. ac.jp/naid/10017095290/en/

Gómez-Caridad, I. \& Bordons, M. (1996) 'Limitaciones en el uso de los indicadores bibliométricos para la evaluación científica'. Política Científica, 46(10), 21-26, ISSN 1133-0554

Gorraiz, J., Gumpenberger, C., \& Glade, T. (2016). On the bibliometric coordinates of four different research fields in Geography. Scientometrics, 107(2), 873-897. https://doi.org/10.1007/s11192-016-1864-y.

Grün B, Hornik K (2011). Topicmodels: An R Package for Fitting Topic Models. Journal of Statistical Software, 40*(13), 1-30. https://doi.org/10.18637/jss.v040.i13 
Gutiérrez, J., \& López-Nieva, P. (2001). Are international journals of human geography really international? Progress in Human Geography, 25(1), 53-69. https://doi.org/10.1191/030913201666823316

Hu, X., Leydesdorff, L., \& Rousseau, R. (2020). Exponential growth in the number of items in the WoS. ISSI News/etter, 16(2), 32-38.

Kanai, J. M., Grant, R., \& Jianu, R. (2018). Cities on and off the map: A bibliometric assessment of urban globalisation research. Urban Studies, 55(12), 2569-2585. https://doi.org/10.1177/0042098017720385

Kleinberg, J. (2003). Bursty and hierarchical structure in streams. Data mining and knowledge discovery, 7(4), 373-397. https://doi.org/10.1023/A:1024940629314

Krugman, P. (1997). Desarrollo, geografía y teoría económica. Antoni Bosch.

Kuhn, T. S. (1970). The structure of scientific revolutions (2nd ed.). The University of Chicago Press.

Méndez Gutiérrez del Valle. R. (1997). Geografía económica. La lógica espacial del capitalismo global. Arial.

Müller, M. (2021). Worlding geography: From linguistic privilege to decolonial anywheres. Progress in Human Geography, https://doi.org/10.1177/0309132520979356.

Ródenas Serra, P., Seguí-Pons, J., \& Ruiz Pérez, M. (2021). A bibliometric analysis of Journal of Transport Geography (1993-2020). Boletín de la Asociación de Geógrafos Españoles (90). https://doi.org/10.21138/bage.3105

Rosenman, E., Loomis, J., \& Kay, K. (2020). Diversity, representation, and the limits of engaged pluralism in (economic) geography. Progress in Human Geography, 44(3), 510-533. https://doi.org/10.1177/0309132519833453

Salvador Oliván, J. A., \& Escolano Utrilla, S. (2018). Comparison of bibliometric indicators in journals of geography indexed in Web of Science. Boletín de la Asociación de Geógrafos Españoles, (76), 131-152. https://doi.org/10.21138/ bage.2518.

Sánchez Hernández, J. L. (2021). El desarrollo teórico de la Geografía Económica en el siglo XXI: hacia la hibridación de los proyectos científicos de la disciplina. Boletín De La Asociación De Geógrafos Españoles, (89). https://doi.org/10.21138/ bage.3080

Unwin, T. (1992): The place of geography, Longman Scientific \& Technical. Londres. (traducción: El lugar de la Geografía, Omega, 1995).

Van Eck, N. J., \& Waltman, L. (2017). Citation-based clustering of publications using CitNetExplorer and VOSviewer. Scientometrics, 111(2), 1053-1070. https://doi.org/10.1007/s11192-017-2300-7

Wang, J., \& Liu, Z. (2014). A bibliometric analysis on rural studies in human geography and related disciplines. Scientometrics, 101(1), 39-59. https://doi.org/10.1007/s11192-014-1388-2.

Zhu, S., Jin, W., \& He, C. (2019). On evolutionary economic geography: a literature review using bibliometric analysis. European Planning Studies, 27(4), 639-660. https://doi.org/10.1080/09654313.2019.1568395 